\title{
Asymptotically safe clockwork mechanism
}

\author{
F. Sannino, ${ }^{1,2}$ J. Smirnov, ${ }^{1}$ and Z. W. Wang ${ }^{1,3, *}$ \\ ${ }^{1} C P^{3}$-Origins, University of Southern Denmark, Campusvej 555230 Odense M, Denmark \\ ${ }^{2}$ SLAC, National Accelerator Laboratory, Stanford University, Stanford, California 94025, USA \\ ${ }^{3}$ Department of Physics, University of Waterloo, Waterloo, Ontario N2L 3G1, Canada
}

(Received 28 February 2019; published 10 October 2019)

\begin{abstract}
In this paper, we demonstrate that safe quantum field theories can accommodate the clockwork mechanism upgrading it to a fundamental theory a lá Wilson. Additionally, the clockwork mechanism naturally sources Yukawa hierarchies for safe completions of the Standard Model. As proof of concept, we investigate a safe Pati-Salam clockwork structure.
\end{abstract}

DOI: 10.1103/PhysRevD.100.075009

The current description of fundamental interactions relies on four-dimensional gauge-Yukawa theories which successfully describe the Standard Model (SM) of particle interactions. However, not all gauge-Yukawa theories are fundamental, i.e., are free from ultraviolet cutoffs. For example, scalar quantum field theories, similar to the one describing the Higgs sector of the SM, or U(1) hypercharge is best viewed as low energy effective field theories. In fact, if one tries to remove the cutoff by pushing it to arbitrary high energies, the resulting consistent quantum field theory corresponds to a noninteracting (trivial) one.

Requiring a given extension of the SM to be nontrivial has proven effective in constraining its interactions and matter content. The Pati-Salam (PS) model of matter field unification [1] is a time-honored example in which one can address the hypercharge triviality issue by embedding it in an asymptotically free theory. From a phenomenological standpoint, it can be commended because it does not induce fast proton decay, and it can even be extended to provide a stable proton [2].

So far, asymptotic freedom has been the well traveled route to resolve the triviality problem. An alternative route is that in which the UV theory acquires an interacting fixed point, before gravity sets in, de facto saving itself from the presence of a cutoff. This unexplored route was opened when the first safe gauge-Yukawa theory was discovered [3]. The proof employed rigorous perturbative methods in the Veneziano-Witten limit that requires a large number of fundamental matter fields and colors with the ratio

\footnotetext{
*Corresponding author. wang@cp3.sdu.dk

Published by the American Physical Society under the terms of the Creative Commons Attribution 4.0 International license. Further distribution of this work must maintain attribution to the author(s) and the published article's title, journal citation, and DOI. Funded by SCOAP.
}

kept fixed. We also learnt that, when loosing asymptotic freedom for the gauge coupling, scalars are essential to drive asymptotic safety via Yukawa interactions in perturbation theory.

To achieve a safe theory with a small number of colors, we needed to go beyond the state-of-the-art of the large number of matter fields techniques $[4,5]$. The first applications of the large $N_{f}$ limit appeared in [6] where it was first explored whether the SM augmented by a large number of vectorlike fermions can have an ultraviolet fixed-point in all couplings. However, only gauge resummation is performed in [6], and thus the final conclusion of achieving a UV fixed point for all the SM couplings is incorrect. The full treatment is studied in [7] and further generalized in [8]. It was found in [7] and later on proved in [8] that while the non-Abelian gauge couplings, Higgs quartic and Yukawa coupling can exhibit a safe fixed point, the hypercharge remains troublesome. In fact, for Abelian theories the fermion mass anomalous dimension diverges at the alleged fixed point [8] suggesting that a safe extension of the SM, like the asymptotically free counterpart, is best obtained by embedding the SM in a non-Abelian gauge structure.

The first non-Abelian safe PS and Trinification embeddings were put forward in $[9,10]$. However, in the minimal models, only one generation of SM fermions can be modeled, since all the Yukawa couplings are determined by the same UV fixed point value with no resulting hierarchy at low energy.

Safe field theories, in the absence of intermediate particle thresholds, are protected against the emergence of a hierarchy problem [11-14]. Nevertheless, when considering phenomenological extensions of the SM, the introduction of vectorlike matter at higher energies, required to provide a safe fixed point, induces a certain degree of finetuning in the scalar sector of the SM.

It is therefore interesting to explore whether one can embed extensions of the SM, aimed at addressing the 
hierarchy problem, into safe quantum field theories. An interesting attempt was considered in [15] where composite extensions of the SM were taken to be safe rather than asymptotically free.

Here, we argue that the clockwork mechanism [16] finds home as four-dimensional safe quantum field theory. This is because concrete realizations of the mechanism require the presence of a large number of new vectorlike fermions that is a natural prediction of safe quantum field theories. As proof of concept, we consider a safe PS structure [9] as natural realization of the clockwork idea. Another benefit of this marriage is the use of the clockwork mechanism to generate Yukawa hierarchies [17].

We will first introduce the model and perform the large number of matter fields renormalization group, we will then demonstrate the presence of safe fixed points and finally offer our conclusions.

\section{A SAFE MODEL}

We first briefly review the PS embedding of the SM suggested [9] and then argue that the extra vectorlike fermions can naturally play the role of clockwork gears and in the process we kill two birds with one stone.

\section{A. Pati-Salam model}

Consider the time-honored PS gauge symmetry group $G_{\mathrm{PS}}[1]$,

$$
G_{\mathrm{PS}}=S U(4) \otimes S U(2)_{L} \otimes S U(2)_{R},
$$

with gauge couplings $g_{4}, g_{L}$, and $g_{R}$, respectively. Here the gauge group $S U(4) \supset S U(3)_{C} \otimes U(1)_{B-L}$, where $S U(3)_{C}$ denotes the SM color gauge group. The SM quark and lepton fields are unified into the $G_{\mathrm{PS}}$ irreducible representations,

$$
\begin{aligned}
\psi_{L i} & =\left(\begin{array}{llll}
u_{L} & u_{L} & u_{L} & \nu_{L} \\
d_{L} & d_{L} & d_{L} & e_{L}
\end{array}\right)_{i} \sim(4,2,1)_{i}, \\
\psi_{R i} & =\left(\begin{array}{llll}
u_{R} & u_{R} & u_{R} & \nu_{R} \\
d_{R} & d_{R} & d_{R} & e_{R}
\end{array}\right)_{i} \sim(4,1,2)_{i},
\end{aligned}
$$

where $i=1,2,3$ is a flavor index. In order to induce the breaking of $G_{\mathrm{PS}}$ to the SM gauge group, we introduce a scalar field $\phi_{R}$ which transforms as the fermion multiplet $\psi_{R}$, that is $\phi_{R} \sim(4,1,2)$,

$$
\phi_{R}=\left(\begin{array}{cc}
\phi_{R}^{u} & \phi_{R}^{0} \\
\phi_{R}^{d} & \phi_{R}^{-}
\end{array}\right)
$$

where the neutral component $\phi_{R}^{0}$ takes a nonzero vev, $v_{R} \equiv$ $\left\langle\phi_{R}^{0}\right\rangle$, such that $G_{\mathrm{PS}} \stackrel{v_{R}}{\rightarrow} S U(3)_{C} \otimes S U(2)_{L} \otimes U(1)_{Y}$. We also introduce an additional (complex) scalar field $\Phi \sim(1,2,2)$, with
TABLE I. Gauge, Yukawa, and scalar quartic couplings of the PS model.

\begin{tabular}{lll}
\hline \hline Gauge & \multicolumn{1}{c}{ Yukawa } & \multicolumn{1}{c}{ Scalar } \\
\hline$S U(4): g_{4}$ & $\psi_{L / R}: y, y_{c}$ & $\phi_{R}: \lambda_{R 1}, \lambda_{R 2}$ \\
$S U(2)_{L}: g_{L}$ & $N_{L}: y_{\nu}$ & Portal: $\lambda_{R \Phi_{1}}, \lambda_{R \Phi_{2}, \lambda_{R \Phi_{3}}}$ \\
$S U(2)_{R}: g_{R}$ & $F: y_{F}$ & $\Phi: \lambda_{1}, \lambda_{2}, \lambda_{3}, \lambda_{4}$ \\
\hline \hline
\end{tabular}

$$
\Phi=\left(\begin{array}{cc}
\phi_{1}^{0} & \phi_{2}^{+} \\
\phi_{1}^{-} & \phi_{2}^{0}
\end{array}\right) \equiv\left(\begin{array}{ll}
\Phi_{1} & \Phi_{2}
\end{array}\right)
$$

which is responsible of the breaking of the EW symmetry.

The most general Yukawa Lagrangian for the matter fields $\psi_{L / R}$ is

$$
\mathcal{L}_{\text {Yuk }}^{\psi}=y \operatorname{Tr}\left[\overline{\psi_{L}} \Phi \psi_{R}\right]+y_{c} \operatorname{Tr}\left[\overline{\psi_{L}} \Phi^{c} \psi_{R}\right]+\text { H.c. },
$$

where $y$ and $y_{c}$ are the Yukawa couplings for the third generation only and are treated as fundamental in this work while the Yukawa couplings of the first two generations will be generated through the clockwork mechanism in the next section.

In the case of a self-conjugate bi-doublet field $\Phi \equiv \Phi^{c}$, one obtains degenerate masses at tree-level, namely

$$
m_{t}=m_{b}=m_{\tau}=m_{\nu_{\tau}} .
$$

In order to separate the neutrino and top masses in Eq. (6), we implement the seesaw mechanism [18-21] by adding a new chiral fermion singlet $N_{L} \sim(1,1,1)$, which has Yukawa interaction (see, e.g., $[9,22]$ for more details),

$$
\mathcal{L}_{\text {Yuk }}^{N}=-y_{\nu} \overline{N_{L}} \operatorname{Tr}\left[\phi_{R}^{\dagger} \psi_{R}\right]+\text { H.c. }
$$

In order to split the mass of top, bottom, and tau lepton in Eq. (6), we introduce a new vectorlike fermion $F \sim$ $(10,1,1)$ with mass $M_{F}$ and Yukawa interactions (see, e.g., $[9,22]$ for more details),

$$
\mathcal{L}_{\text {Yuk }}^{F}=y_{F} \operatorname{Tr}\left(\overline{F_{L}} \phi_{R}^{T} i \tau_{2} \psi_{R}\right)+\text { H.c. }
$$

All the field contents and couplings are summarized in Table I.

\section{B. Clockwork extension}

To realize the clockwork mechanism, we introduce $N_{(i)}$, $(i=1,2)$ pair of vectorlike fermions $\left(Q_{L, 1_{(i)}}, Q_{R, 1_{(i)}}\right), \ldots$, $\left(Q_{L, N_{(i)}}, Q_{R, N_{(i)}}\right)$ with one extra chiral fermion $Q_{R, 0_{(i)}}$ [i.e., each generation $(i)$ of the PS fermions is associated with a clockwork chain with $N_{(i)}$ nodes]. Here $(i)$ denotes the clockwork chain for the first and second generations of the PS (also SM) fermion particles, and for any number of $N_{(i)}$, the chiral fermions $Q_{L, N_{(i)}}$ and $Q_{R, N_{(i)}}$ are charged, 


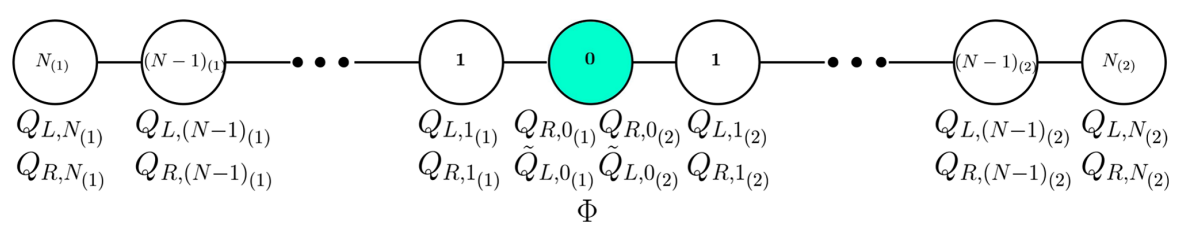

FIG. 1. The diagram summarizes the clockwork chain.

respectively, under the fundamental representation $(4,2,1)$ and $(4,1,2)$ of PS gauge group $G_{P S}=S U(4) \times S U(2)_{L} \times$ $S U(2)_{R}$. In addition, we also introduce $\tilde{Q}_{L, 0_{(1)}}$ and $\tilde{Q}_{L, 0_{(2)}}$ which will only interact, respectively, with the zero node fields $Q_{R, 0_{(1)}}$ and $Q_{R, 0_{(2)}}$ through the Yukawa contributions, i.e.,

$$
\mathcal{L}_{\text {Yuk }}^{Q}=y_{1} \overline{\tilde{Q}}_{L, 0_{(1)}} \Phi Q_{R, 0_{(1)}}+y_{2} \overline{\tilde{Q}}_{L, 0_{(2)}} \Phi Q_{R, 0_{(2)}},
$$

where $\Phi$ is the scalar bi-doublet in the PS model with charge assignment $(1,2,2)$ and $y_{1}, y_{2}$ are the fundamental Yuakwa couplings which have the same value as the Yukawa coupling of the third generation, i.e., $y_{1}=y_{2}=$ $y=y_{c}$. Note that in clockwork type construction of the models, it almost always assumes that the scalar fields are confined to only couple to the fields at one end of the chain where in this work, it is the 0 node. The clockwork mechanism is realized by the following clockwork chain interaction (see also Fig. 1):

$$
\begin{aligned}
\mathcal{L}_{\text {clock }}^{Q_{R}}= & -m_{(1)} \sum_{j=1}^{N_{(1)}}\left(\bar{Q}_{L, j_{(1)}} Q_{R, j_{(1)}}-q_{(1)} \bar{Q}_{L, j_{(1)}} Q_{R, j-1_{(1)}}\right) \\
& -m_{(2)} \sum_{j=1}^{N_{(2)}}\left(\bar{Q}_{L, j_{(2)}} Q_{R, j_{(2)}}-q_{(2)} \bar{Q}_{L, j_{(2)}} Q_{R, j-1_{(2)}}\right),
\end{aligned}
$$

where $q_{(i)}>1,(i=1,2)$ is required to realize the clockwork mechanism. For simplicity, in the following, we assume $q_{(1)}=q_{(2)}=q$ and $m_{(1)}=m_{(2)}=m$ (i.e., all the clockwork vectorlike fermions are introduced at one scale). Actually, when turning on the difference between $q$ and $m$, we will have more freedom and bigger parameter space to explore. After diagonalizing the mass matrix, we obtain $M_{Q_{(i)}}=\operatorname{diag}\left(0, M_{1_{(i)}}, \ldots, M_{N_{(i)}}\right),(i=1,2)$ where there is always one massless mode $\psi_{R, 0_{(i)}},(i=1,2)$. It is intriguing that the massless modes $\psi_{R, 0_{(i)}},(i=1,2)$ overlaps with the fields at the zero node of the chain (see Fig. 1) with a suppression factor, i.e., $\psi_{R, 0_{(i)}}=1 / q^{N_{(i)}} Q_{R, 0_{(i)}}$. Thus, the Yukawa coupling of the $i$ th generations of the PS fermions which originates from the Yukawa interaction terms between $\tilde{Q}_{L, 0_{(i)}}$ and the massless mode $\psi_{R, 0_{(i)}}$ will also be suppressed by $1 / q^{N_{(i)}}$ leading to

$$
\begin{aligned}
\mathcal{L}_{\mathrm{Yuk}}^{\mathrm{eff}} & =y_{1}^{\mathrm{eff}} \overline{\tilde{Q}}_{L, 0_{(1)}} \Phi \psi_{R, 0_{(1)}}+y_{2}^{\mathrm{eff}} \overline{\tilde{Q}}_{L, 0_{(2)}} \Phi \psi_{R, 0_{(2)}} \\
& =\frac{y_{1}}{q^{N_{(1)}}} \overline{\tilde{Q}}_{L, 0_{(1)}} \Phi \psi_{R, 0_{(1)}}+\frac{y_{2}}{q^{N_{(2)}}} \tilde{\tilde{Q}}_{L, 0_{(2)}} \Phi \psi_{R, 0_{(2)}},
\end{aligned}
$$

where it is clear that the effective Yukawa couplings are suppressed and this will be the key to realize the hierarchies of the Yukawa couplings between different generations of the SM fermions. For reasons that will be presented later in Sec. IV, we treat the third generation Yukawa coupling of the PS fermions (i.e., the SM top Yukawa) as the fundamental one, while the first and the second generation Yukawa couplings will be generated through the clockwork mechanism.

\section{LARGE- $N_{F}$ BETA FUNCTIONS}

The beauty of large- $N_{F}$ beta function is by noticing that a subset of the Feynman diagrams (denoted as bubble chain) can be summed up into a closed form at $1 / N_{F}$ order. Thus, all the higher order information up to $1 / N_{F}$ order is encoded in the summation functions denoted as $F_{1}(A), H_{1}(A), H_{0}(A)$ below. It also deserves to note that these summation functions possess the pole structures,

$$
F_{1}(A) \sim \log (1-2 A / 15), \quad H_{1}(A) \sim \log (1-A / 3),
$$

which guarantees the UV fixed point for the gauge beta functions and opens the possibility for the fixed point solutions for all the couplings.

To the leading $1 / N_{F}$ order, the higher order (ho) contributions to the general renormalization group (RG) functions of the gauge couplings are computed in [8], while for the simple gauge groups in $[5,23]$ and for the Abelian in [4]. Here we summarize the results. The ho contributions to $d \alpha_{i} / d \log \mu$ (in the semisimple case) are

$$
\begin{aligned}
\beta_{i}^{\text {ho }} & =\frac{2 A_{i} \alpha_{i}}{3}\left(\frac{d\left(R_{i}\right) H_{1_{i}}\left(A_{i}\right)}{N_{F_{i}} \prod_{k} d\left(R_{\psi}^{k}\right)}+\frac{\sum_{j} d\left(G_{j}\right) F_{1_{j}}\left(A_{j}\right)}{N_{F_{i}} \prod_{k} d\left(R_{\psi}^{k}\right)}\right), \\
\alpha_{i} & \equiv \frac{g_{i}^{2}}{(4 \pi)^{2}}(i=L, R, C),
\end{aligned}
$$

with the functions $H_{1 i}$ and the t'Hooft couplings $A_{i}$, 


$$
\begin{aligned}
A_{i} & =4 \alpha_{i} T_{R} N_{F_{i}} \frac{\prod_{k} d\left(R_{\psi}^{k}\right)}{d\left(R_{\psi}^{i}\right)} \\
H_{1_{i}} & =\frac{-11}{4} \frac{C_{G}}{T_{R}}+\int_{0}^{A_{i} / 3} I_{1}(x) I_{2}(x) d x, \\
F_{1_{j}} & =\int_{0}^{A_{j} / 3} I_{1}(x) d x,
\end{aligned}
$$

where $I_{1}(x)$ and $I_{2}(x)$ are

$$
\begin{aligned}
I_{1}(x)= & \frac{(1+x)(2 x-1)^{2}(2 x-3)^{2} \sin (\pi x)^{3}}{(x-2) \pi^{3}} \\
& \times\left(\Gamma(x-1)^{2} \Gamma(-2 x)\right) \\
I_{2}(x)= & \frac{C_{R}}{T_{R}}+\frac{\left(20-43 x+32 x^{2}-14 x^{3}+4 x^{4}\right)}{4(2 x-1)(2 x-3)\left(1-x^{2}\right)} \frac{C_{G}}{T_{R}} .
\end{aligned}
$$

The Dynkin indices are $T_{R}=1 / 2\left(N_{c_{i}}\right)$ for the fundamental (adjoint) representation while $d\left(R_{\psi}^{k}\right)$ denotes the dimension of the fermion representation.

The RG functions of the (semisimple) gauge couplings are

$$
\begin{aligned}
\beta_{\alpha_{2 L}}^{\text {tot }}= & \frac{d \alpha_{2 L}}{d \log \mu}=\beta_{\alpha_{2 L}}^{\text {lloop }}+\beta_{\alpha_{2 L}}^{\text {ho }}=-6 \alpha_{2 L}^{2} \\
& +\frac{2 A_{2 L} \alpha_{2 L}}{3}\left(1+\frac{H_{1_{2 L}}\left(A_{2 L}\right)}{4 N_{F}}+\frac{15}{8} \frac{F_{1_{4}}\left(A_{4}\right)}{N_{F}}\right) \\
\beta_{\alpha_{2 R}}^{\text {tot }}= & \frac{d \alpha_{2 R}}{d \log \mu}=\beta_{\alpha_{2 R}}^{\text {lloop }}+\beta_{\alpha_{2 R}}^{\text {ho }}=-\frac{14}{3} \alpha_{2 R}^{2} \\
& +\frac{2 A_{2 R} \alpha_{2 R}}{3}\left(1+\frac{H_{1_{2 R}}\left(A_{2 R}\right)}{4 N_{F}}+\frac{15}{8} \frac{F_{1_{4}}\left(A_{4}\right)}{N_{F}}\right) \\
\beta_{\alpha_{4}}^{\text {tot }}= & \frac{d \alpha_{4}}{d \log \mu}=\beta_{\alpha_{4}}^{\text {loop }}+\beta_{\alpha_{4}}^{\text {ho }}=-18 \alpha_{4}^{2} \\
& +\frac{2 A_{4} \alpha_{4}}{3}\left(1+\frac{H_{1_{4}}\left(A_{4}\right)}{4 N_{F}}+\sum_{i=L / R} \frac{3}{16}\left(\frac{F_{1_{2 i}}\left(A_{2 i}\right)}{N_{F}}\right)\right),
\end{aligned}
$$

The Yukawa beta function reads

$$
\begin{aligned}
\beta_{y} & =c_{1} y^{3}+y \sum_{\alpha} c_{\alpha} g_{\alpha}^{2} I_{y}\left(A_{\alpha}\right), \quad \text { with } \\
I_{y}\left(A_{\alpha}\right) & =H_{\phi}\left(0, \frac{2}{3} A_{\alpha}\right)\left(1+A_{\alpha} \frac{C_{2}\left(R_{\phi}^{\alpha}\right)}{6\left(C_{2}\left(R_{\chi}^{\alpha}\right)+C_{2}\left(R_{\xi}^{\alpha}\right)\right)}\right) \\
H_{\phi}(x) & =H_{0}(x)=\frac{\left(1-\frac{x}{3}\right) \Gamma(4-x)}{3 \Gamma^{2}\left(2-\frac{x}{2}\right) \Gamma\left(3-\frac{x}{2}\right) \Gamma\left(1+\frac{x}{2}\right)}
\end{aligned}
$$

containing information about the resumed fermion bubbles and $c_{1}, c_{\alpha}$ are the standard one-loop coefficients for the Yukawa beta function, while $C_{2}\left(R_{\phi}^{\alpha}\right), C_{2}\left(R_{\chi}^{\alpha}\right), C_{2}\left(R_{\xi}^{\alpha}\right)$ are the Casimir operators of the corresponding scalar and fermion fields. Thus, when $c_{1}, c_{\alpha}$ are known, the full Yukawa beta function follows. Similarly, for the quartic coupling, we write

$$
\begin{aligned}
\beta_{\lambda}= & c_{1} \lambda^{2}+\lambda \sum_{\alpha} c_{\alpha} g_{\alpha}^{2} I_{\lambda g^{2}}\left(A_{\alpha}\right)+\sum_{\alpha} c_{\alpha}^{\prime} g_{\alpha}^{4} I_{g^{4}}\left(A_{\alpha}\right) \\
& +\sum_{\alpha<\beta} c_{\alpha \beta} g_{\alpha}^{2} g_{\beta}^{2} \operatorname{Ig}_{g_{1}^{2} g_{2}^{2}}^{\mathrm{tot}}\left(A_{\alpha}, A_{\beta}\right),
\end{aligned}
$$

with $c_{1}, c_{\alpha}, c_{\alpha}^{\prime}, c_{\alpha \beta}$ the known one-loop coefficients for the quartic beta function and the resumed fermion bubbles appear via

$$
\begin{aligned}
I_{\lambda g^{2}}\left(A_{\alpha}\right) & =H_{\phi}\left(0, \frac{2}{3} A_{\alpha}\right) \\
I_{g^{4}}\left(A_{\alpha}\right) & =H_{\lambda}\left(1, \frac{2}{3} A_{\alpha}\right)+A_{\alpha} \frac{d H_{\lambda}\left(1, \frac{2}{3} A_{\alpha}\right)}{d A_{\alpha}} \\
I_{g_{1}^{2} g_{2}^{2}}^{\mathrm{tot}}\left(A_{\alpha}, A_{\beta}\right) & =\frac{1}{3}\left[I_{g_{1}^{2} g_{2}^{2}}\left(A_{\alpha}, 0\right)+I_{g_{1}^{2} g_{2}^{2}}\left(0, A_{\beta}\right)+I_{g_{1}^{2} g_{2}^{2}}\left(A_{\alpha}, A_{\beta}\right)\right] \\
I_{g_{1}^{2} g_{2}^{2}}\left(A_{\alpha}, A_{\beta}\right) & =\frac{1}{A_{\alpha}-A_{\beta}}\left[A_{\alpha} H_{\lambda}\left(1, \frac{2}{3} A_{\alpha}\right)-A_{\beta} H_{\lambda}\left(1, \frac{2}{3} A_{\beta}\right)\right], \\
H_{\lambda}(1, x) & =\left(1-\frac{x}{4}\right) H_{0}(x)=\frac{\left(1-\frac{x}{4}\right)\left(1-\frac{x}{3}\right) \Gamma(4-x)}{3 \Gamma^{2}\left(2-\frac{x}{2}\right) \Gamma\left(3-\frac{x}{2}\right) \Gamma\left(1+\frac{x}{2}\right)} .
\end{aligned}
$$

We therefore have the quartic beta function including the bubble diagram contributions when $c_{1}, c_{\alpha}, c_{\alpha}^{\prime}, c_{\alpha \beta}$ are known.

\section{SAFE CLOCKWORK FIXED POINTS}

The clockwork vectorlike fermions are charged under $G_{P S}$ with the following charge assignment:

$N_{F}(4,1,2) \oplus N_{F}(4,2,1), \quad N_{F}=N_{(1)}+N_{(2)}$.

The physical mass spectrum of the vectorlike fermions ranges from $m(q-1)$ to $m(q+1)$ with $\delta m=2 m$ among the states that it is much smaller than the scale hierarchy between the transition scale of the UV fixed point at around $10^{11} \mathrm{GeV}$ and the electroweak scale at $100 \mathrm{GeV}$. It is therefore reasonable to consider, in first approximation, the two generations of vectorlike fermions $N_{(1)}, N_{(2)}$ to appear at the same scale identified with the PS symmetry breaking scale $(\sim 1000 \mathrm{TeV})$. Thus, above the PS symmetry breaking scale, we use directly $N_{F}$ when analyzing the RG flows. We list the gauge, Yukawa, and scalar couplings in Table I.

For a given value of $N_{F}$, the gauge couplings at the UV fixed point can be treated as background values (i.e., constants in the RG functions of other couplings). This is so because the gauge-coupling UV fixed point depends only on $N_{F}$ and the group structure. Using the one loop beta functions in [9], including the large $N_{F}$ corrections 
TABLE II. It summarizes the UV fixed point solution for $N_{F}=13$ involving the bubble diagram contributions in the Yukawa and quartic RG beta functions. $y_{F}$ is asymptotically free and thus is zero at the fixed point.

\begin{tabular}{lllllllllll}
\hline \hline$\lambda_{1}$ & $\lambda_{2}$ & $\lambda_{3}$ & $\lambda_{4}$ & $\lambda_{R \Phi_{1}}$ & $\lambda_{R \Phi_{2}}$ & $\lambda_{R 1}$ & $\lambda_{R 2}$ & $y$ & $y_{c}$ & $y_{\nu}$ \\
\hline
\end{tabular} \begin{tabular}{llllllllll}
\hline 0.13 & 0.01 & 0.03 & 0.05 & 0.10 & 0.01 & $0.34-0.29$ & 0.53 & 0.53 & 0.67 \\
\hline \hline
\end{tabular}

[i.e., Eqs. (17) and (18)], we solve for $\left\{\beta_{i}=0\right\}$ where $i$ denotes all the Yukawa and scalar couplings in Table I. A sample UV fixed point solution with $N_{F}=13$ is shown in Table II where we have selected the solution to satisfy the vacuum stability conditions. In Fig. 2, we show the RG running of the three gauge couplings that achieve a fixed point after $10^{11} \mathrm{GeV}$. It is pleasing that all gauge couplings assume the same value in the UV due to nature and structure of the fixed point. For the Yukawa couplings and quartic couplings, the RG running of them is similar to the figures shown in Ref. [9]. A minor threshold effect is present before and after involving the vectorlike fermions, but barely visible in the logarithmic plot.

To match onto the SM, we consider the RG flows below the PS symmetry breaking scale. After PS symmetry breaking, the scalar bi-doublet should match the conventional two Higgs doublet model which is defined by the Lagrangian

$$
\begin{aligned}
V_{H}= & m_{11}^{2} \Phi_{1}^{\dagger} \Phi_{1}+m_{22}^{2} \Phi_{2}^{\dagger} \Phi_{2}-\left(m_{12}^{2} \Phi_{1}^{\dagger} \Phi_{2}+\text { H.c. }\right) \\
& +\bar{\lambda}_{1}\left(\Phi_{1}^{\dagger} \Phi_{1}\right)^{2}+\bar{\lambda}_{2}\left(\Phi_{2}^{\dagger} \Phi_{2}\right)^{2}+\bar{\lambda}_{3}\left(\Phi_{1}^{\dagger} \Phi_{1}\right)\left(\Phi_{2}^{\dagger} \Phi_{2}\right) \\
& +\bar{\lambda}_{4}\left(\Phi_{1}^{\dagger} \Phi_{2}\right)\left(\Phi_{2}^{\dagger} \Phi_{1}\right)+\left[\frac{1}{2} \bar{\lambda}_{5}\left(\Phi_{1}^{\dagger} \Phi_{2}\right)^{2}\right. \\
& \left.+\bar{\lambda}_{6}\left(\Phi_{1}^{\dagger} \Phi_{1}\right)\left(\Phi_{1}^{\dagger} \Phi_{2}\right)+\bar{\lambda}_{7}\left(\Phi_{2}^{\dagger} \Phi_{2}\right)\left(\Phi_{1}^{\dagger} \Phi_{2}\right)+\text { H.c. }\right],
\end{aligned}
$$

and the matching conditions with the scalar couplings in Table I are (see [9])

$$
\begin{aligned}
& \bar{\lambda}_{1}=\lambda_{1}, \quad \bar{\lambda}_{2}=\lambda_{1}, \quad \bar{\lambda}_{3}=2 \lambda_{1}, \quad \bar{\lambda}_{4}=4\left(-2 \lambda_{2}+\lambda_{4}\right) \\
& \bar{\lambda}_{5}=4 \lambda_{2}, \quad \bar{\lambda}_{6}=-\lambda_{3}, \quad \bar{\lambda}_{7}=\lambda_{3} .
\end{aligned}
$$

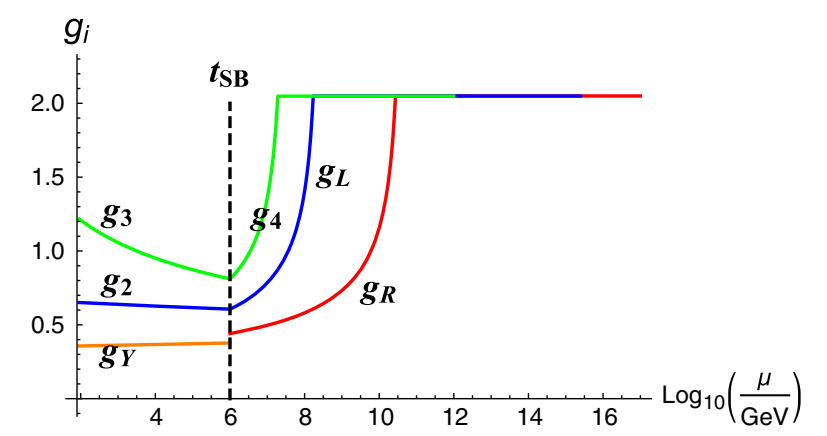

FIG. 2. The diagram shows the running of the gauge couplings.
In our system, when $N_{F}$ is given and the PS symmetry breaking scale is chosen, by using the RG running from the UV fixed point, we obtain the coupling values at the symmetry breaking scale. We can venture below the symmetry breaking scale by using the two Higgs doublet beta functions given in [24]. Implementing the matching conditions Eq. (22), we treat the coupling values obtained at the PS symmetry breaking scale as the new initial conditions. We chose the UV fixed point solution shown in Table II as a starting point and PS symmetry breaking scale at $1000 \mathrm{TeV}$ to find

$\bar{\lambda}_{1}=0.178, \quad \bar{\lambda}_{2}=0.178, \quad \bar{\lambda}_{3}=0.105$,

$\bar{\lambda}_{4}=0.070, \quad \bar{\lambda}_{5}=0.038, \quad y_{\text {top }}=0.987$,

where the couplings are all defined at the electroweak scale, and with a phenomenologically acceptable top Yukawa coupling. The neutral Higgs mass matrix reads

$$
\left[\begin{array}{cc}
\frac{m_{12}^{2} v_{2}}{v_{1}}+2 \bar{\lambda}_{1} v_{1}^{2} & -m_{12}^{2}+\bar{\lambda}_{345} v_{1} v_{2} \\
-m_{12}^{2}+\bar{\lambda}_{345} v_{1} v_{2} & \frac{m_{12}^{2} v_{2}}{v_{1}}+2 \bar{\lambda}_{2} v_{2}^{2}
\end{array}\right],
$$

where $\bar{\lambda}_{345} \equiv \bar{\lambda}_{3}+\bar{\lambda}_{4}+\bar{\lambda}_{5}$. Equation (24) provides a light Higgs mass at $120 \mathrm{GeV}$ and a heavier Higgs mass at $500 \mathrm{GeV}$ when setting $m_{12} \sim 350 \mathrm{GeV}$ (where the lower bound of the heavier $C P$-even scalar mass is around $500 \mathrm{GeV}$; see, e.g., [25]). Note that the scalar mass predictions are $m_{12}$ dependent. When setting $m_{12}=0$, the light Higgs will be massless while the heavy Higgs will be around $121 \mathrm{GeV}$. However, when slightly increasing the $m_{12}$ parameter, the light Higgs will increase correspondingly until $m_{12} \sim 100 \mathrm{GeV}$. After that, the light Higgs mass freezes at around $120 \mathrm{GeV}$, while the heavy Higgs mass keeps increasing with $m_{12}$.

Summarizing for $N_{F}=13$, we can match both the Higgs mass and the top Yukawa coupling at the electroweak scale. We searched the full parameter space in the range of $N_{F} \in$ $(10,200)$ and $N_{F}=13,14$, and the UV fixed point solutions in Table II agree best with the low energy data. We note that $y_{F}$ is asymptotically free for all viable solutions. We have therefore provided a safe clockwork completion of the SM.

\section{LIGHT GENERATIONS AND CONCLUSIONS}

The mass hierarchies among the SM fermion generations are controlled by the clockwork parameter $q$. The relations among $q^{N_{(1)}}, q^{N_{(2)}}$ and the light quark masses are

$q^{N_{(1)}}=\frac{m_{\text {top }}}{m_{u}}, \quad q^{N_{(2)}}=\frac{m_{\text {top }}}{m_{c}}, \quad N_{(1)}+N_{(2)}=13$, 
where $m_{\text {top }}=173 \mathrm{GeV}, m_{c}=1.29 \mathrm{GeV}$, and $m_{u}=2.3 \mathrm{MeV}$. By solving Eq. (25), we find

$$
N_{(1)}=9, \quad N_{(2)}=4, \quad q=3.46 .
$$

A fair point is whether we have enough flavors to argue for the robustness of the large $N_{f}$ expansion. Following [8], the required condition for the large $N_{f}$ expansion is estimated to be $N_{f}>10 N_{c}$ under fundamental representation of $S U\left(N_{c}\right)$. It is derived by comparing the large $1 / N_{f}$ order beta function with the known higher order perturbative results. In the following, we generalize the corresponding lower bound of $N_{f}$ to semisimple gauge group,

$$
\begin{gathered}
S U(4): 2 N_{F} \prod_{k} d\left(R_{\psi}^{k}\right) / d\left(R_{\psi}^{i}\right)=52>10 N_{c}=40 \\
S U(2)_{L}: N_{F} \prod_{k} d\left(R_{\psi}^{k}\right) / d\left(R_{\psi}^{i}\right)=52>10 N_{c}=20 \\
S U(2)_{R}: N_{F} \prod_{k} d\left(R_{\psi}^{k}\right) / d\left(R_{\psi}^{i}\right)=52>10 N_{c}=20,
\end{gathered}
$$

where the extra factor of 2 for $S U(4)$ comes from both leftand right-handed species charged under $S U(4)$. We therefore have that $N_{F}=13$ satisfies the estimated lower bound of the conformal window.

We note that the mass hierarchies of the down-type quark as well as the electrons among different generations need to be induced by additional operators in order to match the SM values. A possibility includes the addition of 10-dim multiplets under the PS symmetry group. Another possibility is to introduce multiple clockwork chains to trigger mass hierarchies with different parameter q. The higher loop order RG running anlaysis may also contribute to the mass splittings. However, a detailed study of these very interesting possibilities goes beyond the scope of the present work.

We have shown that it is mutual beneficial to embed the clockwork mechanism into safe quantum field theories, since the clockwork offers natural ways to generate the observed Yukawa hierarchies while safe field theories naturally predict a large number of vectorlike fields for the clockwork to be operative.

\section{ACKNOWLEDGMENTS}

F. S. acknowledges discussions with Jogesh C. Pati. The work is partially supported by the Danish National Research Foundation under the Grant No. DNRF:90.
[1] J. C. Pati and A. Salam, Phys. Rev. D 10, 275 (1974); 11, 703(E) (1975).

[2] P. Fileviez Perez and S. Ohmer, Phys. Lett. B 768, 86 (2017).

[3] D. F. Litim and F. Sannino, J. High Energy Phys. 12 (2014) 178.

[4] A. Palanques-Mestre and P. Pascual, Commun. Math. Phys. 95, 277 (1984).

[5] J. A. Gracey, Phys. Lett. B 373, 178 (1996).

[6] R. Mann, J. Meffe, F. Sannino, T. Steele, Z. W. Wang, and C. Zhang, Phys. Rev. Lett. 119, 261802 (2017).

[7] G. M. Pelaggi, A. D. Plascencia, A. Salvio, F. Sannino, J. Smirnov, and A. Strumia, Phys. Rev. D 97, 095013 (2018).

[8] O. Antipin, N. A. Dondi, F. Sannino, A. E. Thomsen, and Z. W. Wang, Phys. Rev. D 98, 016003 (2018).

[9] E. Molinaro, F. Sannino, and Z. W. Wang, Phys. Rev. D 98, 115007 (2018).

[10] Z. W. Wang, A. Al Balushi, R. Mann, and H. M. Jiang, Phys. Rev. D 99, 115017 (2019).

[11] G. M. Pelaggi, F. Sannino, A. Strumia, and E. Vigiani, Front. Phys. 5, 49 (2017).

[12] S. Abel and F. Sannino, Phys. Rev. D 96, 056028 (2017).

[13] S. Abel and F. Sannino, Phys. Rev. D 96, 055021 (2017).
[14] S. Abel, E. Mølgaard, and F. Sannino, Phys. Rev. D 99, 035030 (2019).

[15] G. Cacciapaglia, S. Vatani, T. Ma, and Y. Wu, arXiv: 1812.04005.

[16] G. F. Giudice and M. McCullough, J. High Energy Phys. 02 (2017) 036.

[17] R. Alonso, A. Carmona, B. M. Dillon, J. F. Kamenik, J. Martin Camalich, and J. Zupan, J. High Energy Phys. 10 (2018) 099.

[18] P. Minkowski, Phys. Lett. 67B, 421 (1977).

[19] T. Yanagida, Conf. Proc. C 7902131, 95 (1979).

[20] M. Gell-Mann, P. Ramond, and R. Slansky, Conf. Proc. C 790927, 315 (1979).

[21] R. N. Mohapatra and G. Senjanovic, Phys. Rev. Lett. 44, 912 (1980).

[22] R. R. Volkas, Phys. Rev. D 53, 2681 (1996).

[23] B. Holdom, Phys. Lett. B 694, 74 (2010).

[24] G. C. Branco, P. M. Ferreira, L. Lavoura, M. N. Rebelo, M. Sher, and J. P. Silva, Phys. Rep. 516, 1 (2012).

[25] P. Basler, P. M. Ferreira, M. Mühlleitner, and R. Santos, Phys. Rev. D 97, 095024 (2018). 